\title{
Les statuts des ministres du culte dans les États de l'Union européenne
}

Francis Messner

\section{OpenEdition}

12 Journals

Édition électronique

URL : http://journals.openedition.org/rdr/429

DOI : $10.4000 /$ rdr.429

ISSN : 2534-7462

Éditeur

Presses universitaires de Strasbourg

\section{Édition imprimée}

Date de publication : 14 novembre 2019

Pagination : 13-58

ISBN : 979-10-344-0054-6

ISSN : 2493-8637

Référence électronique

Francis Messner, "Les statuts des ministres du culte dans les États de l'Union européenne », Revue du droit des religions [En ligne], 8 | 2019, mis en ligne le 25 novembre 2019, consulté le 20 novembre 2020. URL : http://journals.openedition.org/rdr/429 ; DOI : https://doi.org/10.4000/rdr.429

\section{(c) $(1)(9$}

La revue du droit des religions est mise à disposition selon les termes de la Creative Commons Attribution - Pas d'Utilisation Commerciale 4.0 International - CC BY-NC 4.0. 


\section{LESSTATUTSDESMINISTRESDUCULTE DANSLESÉTATSDEL'UNION EUROPÉENNE}

\section{Francis MESSNER}

Université de Strasbourg / CNRS, Droit, Religion, Entreprise et Société (DRES)

\section{RÉSUMÉ}

Les statuts juridiques des ministres des cultes dans les États européens sont multiples, en raison de la diversité des régimes nationaux des cultes et de la variété des missions des agents cultuels selon les religions. En conformité avec le principe de neutralité de l'État, les règles s'appliquant aux activités des ministres du culte relèvent en général de la compétence des autorités religieuses. Les juges et les pouvoirs publics prennent néanmoins en compte les spécificités de la fonction de ministre du culte et sont amenés à remettre progressivement en question les statuts juridiques forgés au cours de l'histoire, de façon cependant variable selon les États concernés.

\section{Abstract}

The legal status of ministers of religion in the European states is multiple, due to the diversity of church-state relations and the variety of missions entrusted to religious persons from one denomination to another. Pursuant to the principle of state neutrality, the rules applying to the activities of ministers of religion generally fall within the competence of religious authorities. Nevertheless, courts and public authorities take into account the specific characteristics of ministers of religion and are led to gradually question the legal status forged by history, in different ways depending on the states. 
T 'affirmation de l'impérieuse nécessité de l'instauration d'un «statut» des Lministres du culte musulman fait l'objet en France, comme ailleurs en Europe, d'un débat récurrent et le plus souvent mal informé. Partant de ce constat, cette contribution vise à présenter le droit en vigueur dans plusieurs États de l'Union européenne aux fins de déterminer quels sont en ce domaine les points de convergence générés par les évolutions récentes des textes et de la jurisprudence. Les États européens s'accordent certes à reconnaître la compétence exclusive des autorités cultuelles en matière de fixation du statut des ministres du culte (1). Ils divergent toutefois quant aux mécanismes à mettre en place pour prendre en compte les spécificités de leur mission spirituelle (2).

\section{LA FIXATION DU STATUT DES MINISTRES DU CULTE : UNE COMPÉTENCE DES AUTORITÉS CULTUELLES}

La comparaison des statuts juridiques des ministres des cultes au sens large du terme est complexe en raison du caractère différencié des régimes des cultes des États de l'Union européenne d'une part et de la grande diversité de fonctions des agents cultuels des différentes religions d'autre part. Dans la plupart des États européens, les règles s'appliquant aux activités des ministres du culte relèvent des seules autorités religieuses, et cela en conformité avec le principe de neutralité de l'État. Mais dans certains rares cas, le ministre du culte de religions majoritaires est un fonctionnaire de l'État ou encore le titulaire d'un bénéfice, c'est-à-dire usufruitier d'un ensemble de biens servant à sa subsistance. De plus, le ministre du culte est, selon les groupements religieux, un prêtre exerçant un sacerdoce, seul habilité à pouvoir pratiquer des rites sacrés, un conseiller moral, un guide spirituel ou encore un théologien, un prédicateur, un «docteur de la loi » ou un permanent exerçant un métier relationnel fait d'animation, d'écoute et d'accompagnement des personnes. Il combine souvent toutes ces activités. À ces fonctions liées à une conception de la recherche de sens liée à la transcendance, il convient d'ajouter de nouvelles formes de «ministères» encore peu développées, articulées à une recherche de sens adossée à l'immanence. Les humanistes ou la laïcité organisée en Belgique bénéficient, depuis une réforme de la Constitution belge en 1993, des mêmes soutiens que les cultes reconnus dont les ministres sont stipendiés ${ }^{1}$. Ils sont assimilés à ces derniers pour tout ce qui concerne la rémunération de leurs permanents.

1. Art. 181 de la Constitution du Royaume de Belgique: « $\S 1^{\text {er }}$. Les traitements et pensions des ministres des cultes sont à la charge de l'État; les sommes nécessaires pour y 
Contrairement à une idée encore largement reçue, l'État, tenu de respecter le principe de neutralité en matière religieuse et la liberté d'organisation ou l'autonomie des cultes, ne peut définir le statut des ministres du culte. Cette qualification relève en principe de la seule compétence des autorités des religions, même s'il existe des exceptions comme dans certains pays nordiques où les pasteurs sont des fonctionnaires ${ }^{2}$. Les pouvoirs publics se contentent en règle générale de tirer les conséquences de la définition donnée par les autorités religieuses, ce qui suppose cependant qu'ils en connaissent les éléments. Les religions organisées disposent dans la majorité des cas d'un corpus de textes déterminant les formes de régulation normative de leurs institutions, activités et personnels. Ainsi, le code de droit canonique de l'Église catholique précise le statut des prêtres, des diacres et des laïcs en mission pastorale. Il intègre les conditions d'âge, de formation «humaine et scientifique», d'accession à une charge pastorale, c'est-à-dire à un office dans un diocèse ou une paroisse, de rémunération, de protection sociale et de révocation. Le contenu du cahier des charges est fixé dans une «lettre de mission ». En France, les conditions d'accès au pastorat de l'Église protestante unie de France (EPUdF) sont précisées dans la constitution de cette Église qui met l'accent sur la formation et le suivi professionnel. Un candidat au pastorat doit être titulaire d'un master de théologie et avoir fait son proposanat, c'est-à-dire un stage. S'il remplit les conditions, il est admis et inscrit au rôle des pasteurs et peut alors être recruté par une paroisse. La procédure de révocation, qui comprend des possibilités de recours, est fixée par la même constitution. Le statut des rabbins et des grands rabbins relève en France, pour le judaïsme consistorial, du règlement général du corps rabbinique du consistoire central, adopté par l'assemblée générale du consistoire central le 16 mars 1975. À l'instar de l'EPUdF, où les pasteurs doivent être inscrits au rôle avant d'être nommés, les rabbins sont tenus de faire partie du «corps rabbinique» et donc d'être qualifiés par l'autorité centrale avant leur nomination. Le corps rabbinique constitue le vivier des rabbins, qui sont de nationalité française et jouissent des droits civils et civiques. Les candidats sont titulaires du diplôme de rabbin délivré par le consistoire central après

faire face sont annuellement portées au budget.

$\S 2$. Les traitements et pensions des délégués des organisations reconnues par la loi qui offrent une assistance morale selon une conception philosophique non confessionnelle sont à la charge de l'État; les sommes nécessaires pour y faire face sont annuellement portées au budget.»

2. Dans la tradition luthérienne, l'Église, qui ne dispose pas toujours d'un droit ecclésial, peut être organisée par l'État. Des États comme la Suède ont cependant mis fin à ce système. V. L. Friedner, «Sweden», in G. Robbers and C. Durham (eds), Encyclopedia of Law and Religion, Leiden, Brill, 2016, p. 404. 
avoir suivi une formation dans le cadre du Séminaire israélite de France ${ }^{3}$ qui lui est institutionnellement lié.

Contrairement au christianisme et au judaïsme, il n'existe pas pour les communautés musulmanes de statut des cadres religieux musulmans défini par les autorités religieuses et accepté par la majeure partie des groupements religieux. Les juristes musulmans ont certes élaboré au cours des siècles une image idéale de l'imam qui relève plus de la morale et de l'idéal de perfection que de la régulation normative. Le corpus des textes fondateurs de l'islam relatif aux imams, au sens du droit musulman, n'aborde guère les aspects organisationnels et statutaires proprement dits. Mais il ne fait pas obstacle à l'adoption d'un modèle facilitant une intégration sociale et juridique. Les États musulmans ont d'ailleurs réglementé le statut de l'imam sans qu'il soit par ailleurs codifié en droit musulman, contrairement aux États européens où, conformément au principe de neutralité, les statuts des imams sont en principe fixés par les cultes. Ainsi, en Turquie, tout comme au Maroc, la fonction d'imam est organisée par l'État dans le cadre d'un statut qui précise sa fonction, son cahier de charges, les modes de recrutement, de cessation de fonction et de rémunération. Il n'existe certes pas dans les textes fondateurs de la religion musulmane de dispositions contraignantes relatives à l'instauration d'un statut, mais rien n'empêche sa création. Ce sont plutôt les circonstances et la volonté politique qui l'imposent. Même l'Arabie saoudite, où le pouvoir religieux s'oppose à la codification du droit musulman, a conféré un statut à l'imam. Mais ces exemples ne sont pas forcément des modèles opérationnels pour les États européens. La séparation entre le temporel et le spirituel n'est pas aussi nette dans les États sociologiquement musulmans, où l'administration étatique des cultes fait parfois fonction d'autorité religieuse, alors que les États européens sont tenus de respecter le principe de neutralité en matière religieuse.

Ceux-ci ont manifesté, après une période d'hésitation, la volonté d'inclure l'islam dans leur régime des cultes ${ }^{4}$, ce qui constitue à la fois une intégration sociale, promeut l'égalité en matière cultuelle et facilite la prise en compte de l'organisation cultuelle particulière à la religion musulmane. Cette démarche

3. Le séminaire israélite de France qui est un établissement supérieur d'enseignement privé déclaré, a été créé en 1829 par l'État et financé par les pouvoirs publics en 1831. Il relève depuis la loi du 9 décembre 1905 du consistoire central israélite.

4. Les États européens ont créé des cadres juridiques en vue de faciliter l'organisation et l'activité des cultes, à l'instar de la loi du 9 décembre 1905 en France ou par le biais de conventions comme en Italie. Les communautés musulmanes ne sont que très rarement organisées dans le cadre de ces régimes des cultes. 
suppose que la religion soit «connue». Elle comprend de manière générale la rédaction d'un statut à destination des ministres du culte ${ }^{5}$ de cette religion et par cette religion, ce qui est le cas pour les religions dites historiques. Les pouvoirs publics sont cependant confrontés à l'absence d'une organisation structurée et homogène des communautés musulmanes et surtout à l'interventionnisme des États d'origine des membres de ces communautés. Il est illustré par l'envoi d'imams fonctionnaires détachés qui ont un statut fixé par leur pays d'origine.

Les imams sont souvent des bénévoles, des fonctionnaires envoyés par des États étrangers ${ }^{6}$ ou encore des salariés occupant à la fois des fonctions d'animation culturelle et des fonctions cultuelles. La quasi-totalité de ces personnels ne relèvent pas encore, ou alors très partiellement, des aménagements prévus par les régimes des cultes qui sont en règle générale caractérisés par l'exclusion du contrat de travail ou par un statut d'agent public sui generis à destination des ecclésiastiques.

Si le statut des ministres du culte au sens large du terme relève, en principe, de la compétence des autorités religieuses, les pouvoirs publics en Europe tiennent cependant compte du caractère particulier de leur fonction dans le cadre de leurs régimes nationaux des cultes.

\section{SALARIAT OU ENGAGEMENT RELIGIEUX? LA PRISE EN COMPTE DES SPÉCIFICITÉS DE LA MISSION SPIRITUELLE ET PASTORALE DES MINISTRES DU CULTE}

La prise en compte des spécificités de la fonction de ministre du culte par les pouvoirs publics en Europe varie en fonction des différents régimes nationaux des cultes et de leur identité historique. Des exemples significatifs comme le régime des Églises nationales, le système des cultes reconnus, celui de droit conventionnel et enfin des systèmes minimalistes adossés aux droits fondamentaux sont certes à même de souligner les différences, mais

5. Notons que, dans la plupart des cas, les communautés musulmanes acceptent, souhaitent ou revendiquent l'établissement d'un statut qui contribue à leur visibilité et à leur intégration dans la société.

6. Près de 300 imams détachés turcs ou algériens exercent leur fonction en France. Une trentaine est par ailleurs rémunérée par le gouvernement marocain sous la forme d'une ligne budgétaire versée à une fédération établie en France. V. N. Goulet et A. ReICHARDT, De l'Islam en France à un Islam de France, établir la transparence et lever les ambiguïtés. Rapport d'information, Sénat, 5 juill. 2016, nº 757. 
sont également en capacité de déterminer des convergences susceptibles de forger un certain nombre de principes communs pour la prise en compte des particularités religieuses liées à la fonction de ministre du culte.

\subsection{BÉNÉFICE ET FONCTIONNARIAT : ÉVOLUTION DES MODÈLES}

Les prêtres anglicans de l'Église établie d'Angleterre, et plus particulièrement les curés (vicars) sont en règle générale titulaires d'un bénéfice ${ }^{7}$. Leur statut est fixé par le droit canonique anglican et plus précisément par le Clergy discipline measure. Le droit canonique anglican fait partie du droit anglais et est interprété, le cas échéant, non seulement par les tribunaux ecclésiastiques, mais également par les tribunaux étatiques ${ }^{8}$. En s'appuyant sur cet exemple propre à l'Église établie, les tribunaux civils anglais estiment de manière récurrente que les ministres du culte en général ne sont pas des salariés et ne sont pas titulaires d'un contrat de travail. Ils perçoivent une indemnité considérée comme un don divin, versée par leur Église en vue d'assurer leur existence matérielle et de permettre la mise en œuvre de leurs activités spirituelles. Celles-ci découlent d'un rite religieux, l'ordination, qui est un évènement à caractère cultuel sans lien avec une relation contractuelle. Ce raisonnement a ainsi été appliqué à un officier de l'Armée du salut dans une décision de $1937^{9}$. À la fin du $x^{e}$ siècle, des juges ont cependant considéré qu'un laïc anglican, donc un agent non ordonné de l'Église, titulaire d'un office sans bénéfice, mais exerçant une activité pastorale, peut être titulaire d'un contrat de travail ${ }^{10}$. Cette affaire a été réglée avant d'être jugée en appel. Par contre dans l'affaire Preston concernant un pasteur révoqué par son Église, le tribunal du travail s'est déclaré incompétent, les ministres du culte n'étant pas des salariés. La cour d'appel a au contraire considéré que Preston était un salarié titulaire d'un contrat de

7. Le bénéfice ecclésiastique était constitué de la dîme, et souvent d'un patrimoine immobilier. Son titulaire percevait une rente, fruit du revenu de ces droits et biens. Actuellement, les biens de l'Église d'Angleterre (patrimoine ecclésiastique: biens meubles et immeubles, produit du rachat des dîmes par la Couronne) sont centralisés. Les titulaires d'un bénéfice perçoivent une part fixe des revenus d'un fonds commun.

8. Le droit canonique anglican fait partie du droit national anglais. À ce titre, il doit se conformer aux principes du droit commun anglais: N. DoE, Canon Law in the Anglican Communion, Oxford University Press, 1998.

9. Roger v Booth, [1937] 2 All ER 751.

10. Il s'agit d'un reader de l'Église d'Angleterre dont la fonction est équivalente à celle d'un laïc coopérateur de la pastorale en France: Barthorpe $v$ Exeter Diocesan Board of Finance [1979] ICR 900. 
travail $^{11}$. Cette position n'a finalement pas été retenue par la Cour suprême qui estime que la cour d'appel n'a pas suffisamment pris en compte la constitution de l'Église méthodiste qui sert de fondement à la relation de Preston avec cette Église. Pour la Cour, le juge ne doit pas traiter les ministres du culte comme une catégorie abstraite sous le poids de la tradition. Il s'agit au contraire d'examiner chaque cas et de prendre en considération les pratiques et les règles fixées par chaque religion, ainsi que les aménagements spécifiques instaurés pour chaque ministre du culte. La Cour suprême écarte ainsi toute présomption d'exclusion du contrat de travail des ministres du culte et recommande d'examiner avant tout les conditions de leur embauche, les règles encadrant leur service et l'intention des parties ${ }^{12}$. Ce qui doit être retenu par le juge n'est pas l'activité du ministre du culte en tant que telle, mais les statuts encadrant et précisant la teneur de son activité et de son engagement au sein de la religion concernée ${ }^{13}$.

La situation du Danemark est relativement différente dans la mesure où le statut des pasteurs de l'Église luthérienne nationale du Danemark ${ }^{14}$ (Folkekirke) est fixé par l'État. En effet, les pasteurs de l'Église nationale sont des fonctionnaires du royaume. Ils relèvent à ce titre du statut général de la fonction publique qui prévoit cependant des aménagements particuliers pour les agents cultuels. En tant que fonctionnaires, ils sont tenus à la loyauté envers l'État et ont la possibilité de se syndiquer. Recrutés par les conseils paroissiaux, les pasteurs de l'Église nationale sont révoqués selon la même procédure que les autres fonctionnaires pour tout ce qui concerne les aspects temporels de leur fonction. Par contre, les aspects spirituels dont font partie la doctrine et la prédication, mais non leur conduite morale ${ }^{15}$, sont de la compétence de juridictions spécialisées. Ces juridictions, dites «des pasteurs et des évêques» interviennent lorsque les croyances fondamentales de l'Église populaire sont mises à mal, notamment lors des prêches ${ }^{16}$. Le rôle de juge

11. President of the Methodist Conference v Preston [2011] EWCA Civ 1581.

12. Ibid.

13. V. N. Doe, «Law and Religion in the Workplace», in M. Rodriguez Blanco (ed.), Law and Religion in the Workplace, Granada, Comares, 2016, p. 393.

14. L'Église évangélique luthérienne est, conformément à l'article 4 de la Constitution du Royaume du Danemark, l'Église nationale danoise. Son statut est fixé par la loi. Elle jouit, comme telle, du soutien de l'État. Il ne s'agit pas d'une Église d'État stricto sensu. L'État danois est neutre en matière religieuse et garantit la liberté de conscience, la liberté individuelle de religion et la liberté d'exercice du culte.

15. Les préférences sexuelles et les situations matrimoniales n'interfèrent pas dans le recrutement d'un pasteur et ne peuvent pas être invoquées comme une cause de licenciement.

16. Citons également le cas d'un pasteur qui refuse de baptiser les enfants au motif que seul le baptême des adultes est théologiquement valable ou encore l'exemple d'un pasteur proclamant la non-existence de Dieu. 
spirituel revient alors aux juridictions judiciaires de première instance qui se transforment en "juridictions des pasteurs» grâce à l'apport de deux experts en théologie qui rejoignent les magistrats. Le jugement de première instance peut être attaqué en appel devant une cour d'appel qui comprend alors trois experts en théologie. Ces experts sont des pasteurs ordonnés ou des théologiens désignés au sort par le biais d'une liste établie par le ministère des Affaires ecclésiastiques en accord avec l'organisation ecclésiastique. Quand l'affaire concerne un évêque, elle est directement portée devant la cour d'appel et la Cour suprême peut être saisie en appel ${ }^{17}$. Même si l'Église populaire, qui est intégrée dans l'appareil administratif de l'État danois, ne dispose pas d'une large autonomie, des mécanismes ont donc été créés en vue de garantir sa liberté d'organisation, notamment en matière doctrinale, par le biais des juridictions de pasteurs.

Les ministres des cultes de toutes les autres religions, et donc de la religion musulmane, relèvent du droit individuel et collectif du travail et, contrairement aux membres de l'Église nationale, bénéficient d'une pleine liberté d'organisation. La loi relative à la protection contre le licenciement (343/1990) n'interdit pas aux employeurs des institutions cultuelles d'exiger que le ministre du culte soit obligatoirement membre de la religion concernée. Le droit danois relatif aux ministres du culte ne s'éloigne donc guère du droit commun: fonctionnariat légèrement aménagé pour les pasteurs de l'Église populaire et salariat d'une entreprise d'éthique pour les ministres des autres religions, y compris pour les ministres du culte musulman.

Le statut des pasteurs de l'Église populaire danoise semble être gravé dans le marbre. Pourtant rien n'est immuable. Ainsi en Suède, la séparation, ou plutôt la sortie de l'Église luthérienne de Suède de l'appareil administratif ${ }^{18}$ a eu pour effet de banaliser le statut des pasteurs qui sont dorénavant traités comme les autres ministres du culte, c'est-à-dire comme tous les autres salariés. En effet, les tribunaux du travail sont compétents pour les litiges concernant les ministres du culte et les autorités religieuses peuvent prendre parallèlement des sanctions en application de leur «discipline». Elles ont notamment la capacité de retirer l'ordination à un pasteur, qui en principe continue d'être salarié de la paroisse employeur mais sans pouvoir exercer les fonctions découlant de l'ordination par l'Église luthérienne de Suède. Le licenciement du pasteur sans ordination est alors possible pour des raisons économiques, lorsque la paroisse n'est pas en mesure d'assurer un salaire

17. L. Christoffersen, «Denmark», in G. Robbers and C. Durham (eds), op. cit., p. 97.

18. L. Friedner, «Sweden», op. cit., p. 404. 
à deux ministres du culte ${ }^{19}$. Avant la «séparation», les pasteurs de l'Église luthérienne étaient embauchés conformément au droit commun du travail et les questions liées au statut spirituel ou cultuel de ces personnels relevaient des juridictions étatiques et non de l'autorité religieuse. L'autonomie des cultes est désormais garantie par le nouveau statut de l'Église luthérienne introduit par la loi sur l'Église de Suède de 1998 et par les textes subséquents. Les pasteurs de l'Église luthérienne de Suède sont certes titulaires d'un contrat de travail, mais tout ce qui relève du cultuel et du spirituel est désormais de la seule compétence des autorités religieuses et ne fait plus partie du contrat de travail. Certaines particularités ont cependant été maintenues. En effet, si la loi sur l'égalité entre les hommes et les femmes prévoit des dérogations pour les cultes non luthériens et notamment pour les catholiques, les musulmans et les orthodoxes, l'ancienne Église nationale qui accepte l'épiscopat féminin relève en cette matière explicitement du droit commun ${ }^{20}$.

Si le système des cultes reconnus dans l'État du Grand-Duché du Luxembourg n'était pas assimilable à celui d'une Église nationale, l'organisation des cultes était intégrée dans l'administration publique et les ministres des cultes reconnus assimilés à des fonctionnaires. Or, la sortie du système des cultes reconnus ${ }^{21}$ par le biais de l'instauration d'un régime des cultes conventionnés a profondément modifié le statut des ministres du culte qui relèvent désormais du droit privé 22 et sont rémunérés par leurs cultes d'appartenance, en partie grâce à des subventions versées par l'État. En cas de conflit, le tribunal du travail était et est toujours compétent ${ }^{23}$ pour l'ensemble des ministres du culte, sans que par ailleurs, neutralité oblige, il puisse s'immiscer dans ce qui relève du spirituel. Il ne peut pas par exemple s'opposer à la décision de révocation d'un clerc jugé «hérétique» par l'autorité religieuse.

19. L. Friedner, «Law and Religion in the Workplace: Sweden », in M. Rodriguez Blanco (ed.), op.cit., p. 389.

20. Ibid.

21. V. F. MESSNER, «Luxembourg: la rationalisation progressive d'un régime des cultes (18011997-2015)», in Mélanges en l'honneur du doyen Jean-Pierre Machelon, Paris, LexisNexis, 2015, p. 777.

22. Convention entre l'État du Grand-Duché de Luxembourg et les communautés religieuses établies au Luxembourg, art. 4: «Les communautés religieuses s'engagent à ne plus recruter leurs collaborateurs à charge du budget de l'État à partir de la date de l'approbation de la présente convention. À partir de cette date, tous les collaborateurs recrutés par une communauté religieuse seront engagés sous un régime de droit privé.»

23. K. PAPAStathis and Ph. Poirier, «Religion at the Workplace: Luxembourg», in M. RodriGUEZ BlANCo (ed.), op. cit., p. 290. 


\subsection{LE PRAGMATISME DU SYSTÈME AUTRICHIEN DES CULTES RECONNUS}

Le régime autrichien des sociétés religieuses, qui est un système de cultes reconnus, tente de concilier la neutralité de l'État, l'autodétermination des cultes et le droit des individus, fussent-ils ministres du culte. Les cultes reconnus, dont l'islam, sont organisés dans le cadre des corporations de droit public. Le statut des ministres du culte au regard du droit du travail est très pragmatique. Il n'y a pas par principe d'exclusion du contrat de travail ou du recours devant une juridiction étatique. Les ministres du culte peuvent saisir une juridiction civile, mais les tribunaux étatiques ne sont pas compétents pour tout ce qui concerne les affaires propres aux communautés religieuses, comme le déplacement d'un ministre du culte pour des raisons disciplinaires «de nature interne ${ }^{24}$, et cela en dépit de l'existence d'un contrat de travail. L'autodétermination des cultes est ainsi garantie. Par contre, un ministre du culte discriminé car ne percevant pas la même rémunération que ses confrères peut légitimement déposer un recours devant un tribunal civil ${ }^{25}$.

Deux communautés islamiques sont reconnues par l'État conformément à la loi sur l'islam de $2015^{26}$ et organisées dans le cadre d'une corporation de droit public: la communauté sunnite et la communauté alévie (chiite). Les statuts de leurs imams et muftis sont fixés par les règlements de ces deux communautés. La constitution de la communauté religieuse islamique sunnite détaille les différentes catégories de ministres du culte, c'est-à-dire les agents religieux dont la fonction est spirituelle et pastorale. Il s'agit des imams, des prédicateurs et des aumôniers. La formation des imams et prédicateurs est de la compétence des autorités religieuses. Par contre, les aumôniers musulmans des établissements publics doivent être titulaires d'une licence en théologie ou d'un diplôme jugé équivalent aux fins d'exercer leur fonction $^{27}$. En Autriche, l'autonomie des cultes n'écarte pas tout contrôle sur les activités et personnels religieux.

24. VfGH 28.11.2011, B 1220/11.

25. OGH 26.11.1974, 4 Ob 41/74.

26. F. MeSSNER, «La loi de 2015 sur l'islam en Autriche», Revue du droit des religions, $\mathrm{n}^{\circ} 2$, 2016, p. 177.

27. Les professeurs de religion des établissements publics et privés et les aumôniers des établissements publics sont obligatoirement formés par la faculté de théologie musulmane de l'Université de Vienne 


\subsection{LES MINISTRES DES CULTES DANS LES SYSTÈMES CONCORDATAIRES OU DE CONVENTIONNEMENT}

La connaissance du statut des ministres du culte occupe une place centrale dans les systèmes de conventionnement, à la différence du clergé fonctionnarisé des Églises nationales où en l'absence d'autonomie des cultes le statut était ou est encore fixé par l'Administration.

En Espagne, l'accord sur les questions juridiques entre le Royaume d'Espagne et le Saint-Siège de janvier 1979 garantit de manière extensive l'autonomie de l'Église catholique ${ }^{28}$. Elle peut s'organiser librement et par voie de conséquence fixer librement le statut des prêtres exerçant une charge paroissiale ou une fonction spirituelle. L'accord de 1979 ne définit pas le ministre du culte catholique et renvoie implicitement au droit canonique le soin de déterminer le statut des clercs de la religion catholique. Or le droit ecclésial catholique n'est pas en principe méconnu des juristes, et donc des juges et des hauts fonctionnaires, puisqu'il constitue une matière à option ${ }^{29}$ dans la plupart des facultés de droit des universités publiques et privées espagnoles ${ }^{30}$, ce qui permet aux autorités publiques de connaître le statut des prêtres catholiques. Ce n'est pas le cas de l'Église protestante et des religions juive et musulmane qui ont également signé des accords avec le gouvernement espagnol en 1992. Les religions non catholiques ont pour ce faire accepté d'identifier et de préciser dans des conventions bilatérales les fonctions ou le cahier des charges d'un ministre du culte, de manière à préserver la neutralité de l'État tout en l'informant sur leur organisation. En effet, il n'appartient pas aux pouvoirs publics de définir le statut des ministres $\mathrm{du}$ culte. Ce sont donc les communautés religieuses qui s'acquittent de cette tâche dans le cadre d'une négociation et d'une collaboration avec l'État. La loi du 12 novembre 1992 approuvant l'accord signé avec les communautés israélites d'Espagne dispose ainsi que le rabbin est « une personne physique exerçant des fonctions religieuses de façon stable et permanente». Ce statut

28. Art. $1^{\text {er }}$ : «L'État espagnol reconnaît à l'Église catholique le droit d'exercer sa mission apostolique et lui garantit l'exercice libre et public des activités qui lui sont propres et spécialement des activités de culte, de juridiction et de magistère».

29. La place de l'enseignement du droit canonique tend cependant à se réduire au sein des universités espagnoles, et cela au profit d'autres matières juridiques. On assiste également au développement, certes modeste, de cours présentant les disciplines des religions protestantes, musulmane et juive.

30. J. M. González del Valle, A. Hollerbach (eds), The Teaching of Church-State Relations in European Universities = L'enseignement du droit ecclésiastique de l'État dans les universités européennes, Leuven, Peeters, 2005. 
est attesté par un certificat délivré par les autorités de la communauté juive signataire de l'accord avec l'État ${ }^{31}$. La définition du responsable de communauté et de l'imam par la loi du 10 novembre 1992 approuvant l'accord avec la Fédération des communautés musulmanes est un peu plus détaillée. Les responsables de communauté et les imams sont des personnes physiques exerçant de manière stable la direction de la prière, la formation et l'assistance spirituelle islamique. Ils sont titulaires d'un certificat délivré par les autorités de la communauté musulmane ${ }^{32}$. Enfin, la loi du 10 novembre 1992 approuvant l'accord avec la Fédération des Églises évangéliques d'Espagne (FEREDE) précise que les ministres du culte des Églises membres sont des personnes physiques qui se consacrent de manière stable aux fonctions de culte ou d'assistance religieuse et sont accréditées par le biais de certifications délivrées par les communautés locales sous le sceau de la commission permanente de la FEREDE. La certification est indispensable car elle permet d'identifier l'agent cultuel autorisé à célébrer un mariage religieux produisant des effets civils ${ }^{33}$. Mais pour l'Église catholique, il n'existe pas de certification des prêtres, l'article 6 de l'accord de 1979 se contentant de préciser que «L'État reconnaît les effets civils du mariage célébré selon les règles du droit canonique». Les éléments pivots de la définition contenue dans les accords, une fonction stable et la certification par la communauté religieuse concernée, sont également appliqués par les pouvoirs publics aux ministres des religions non conventionnées lors de leur inscription dans le registre des entités religieuses ${ }^{34}$.

La Cour suprême exclut l'existence d'un contrat de travail pour l'ensemble des ministres du culte. Dans une affaire concernant un décret de 1999

31. Le certificat est délivré par la communauté locale sous le sceau des responsables de la Fédération des communautés juives (FCI).

32. Le certificat est délivré par la communauté locale sous le sceau de responsables de la Commission islamique d'Espagne (CIE).

33. Par ex., loi du 12 novembre 1992 approuvant l'accord signé avec les communautés israélites d'Espagne, art. 7: «Sont reconnus les effets civils du mariage célébré selon les procédures du droit israélite devant les ministres du culte des communautés appartenant à la Fédération des communautés israélites d'Espagne. Pour la pleine reconnaissance de ces effets, l'inscription du mariage au registre civil sera nécessaire».

34. Loi organique du 5 juillet 1980 sur la liberté religieuse, art. 5: «1. Les Églises, confessions et communautés religieuses et leurs fédérations jouiront de la personnalité juridique une fois inscrites au registre public correspondant, qui sera créé à cet effet au Ministère de la Justice.

2. L'inscription s'effectuera au moyen d'une demande, accompagnée d'un document faisant foi de leur fondation ou établissement en Espagne, fins religieuses, dénomination et autres données d'identification, règles de fonctionnement et organes représentatifs, avec l'indication de leurs facultés et des conditions requises pour leur désignation valable.» 
relatif à l'affiliation des pasteurs de la Fédération des Églises évangéliques d'Espagne au régime général de la sécurité sociale, avec une exclusion de relations contractuelles entre le ministre du culte et l'entité religieuse, la Cour suprême, dans une décision du 4 juin $2001^{35}$, a considéré qu'il était impossible d'instaurer un contrat de travail au bénéfice de l'agent cultuel. Le juge doit tenir compte des relations spécifiques entretenues par les deux partenaires. Elles s'opposent selon lui, et cela conformément à la loi sur la liberté religieuse de 1980 qui permet aux cultes de fixer le statut de leurs ministres, à la possibilité de faire un choix pour ces communautés religieuses entre l'exclusion ou l'instauration d'un contrat de travail. Cette décision a été vivement critiquée par la doctrine qui l'a qualifiée de «tautologique ${ }^{36}$ ». Le modèle historico-canonique du ministre du culte catholique reste cependant très prégnant.

Les principes régissant les relations sui generis entre les ministres du culte et les autorités religieuses en Italie sont similaires à ceux qui s'imposent en Espagne. En effet, l'article 7 de la Constitution italienne, tout comme l'article premier des accords de Villa Madame ${ }^{37}$, disposent que «L'État et l'Église catholique sont, chacun dans leur domaine, indépendants et souverains » et l'article 8 de la Constitution précise que «Les religions autres que la religion catholique ont le droit de s'organiser selon leurs propres statuts, à condition qu'ils ne soient pas en contradiction avec l'ordre juridique italien ». Or, le droit canonique de l'Église catholique qui fixe le statut des clercs est à l'instar de l'Espagne enseigné dans les facultés de droit des universités italiennes et devrait par voie de conséquence être connu des juristes. Il n'est donc pas détaillé dans les accords de Villa Madame. Les accords passés avec les autres religions stipulent que leurs ministres des cultes sont accrédités par les autorités religieuses. Cette accréditation est nécessaire dans le système $\mathrm{du}$ droit ecclésiastique ${ }^{38}$ italien. Elle permet notamment aux ministres du culte bénéficiant de cette reconnaissance d'occuper des postes d'aumôniers dans les services publics et de célébrer des mariages religieux produisant des effets civils ${ }^{39}$. Mais, contrairement aux accords espagnols, les fonctions des

35. Cour constitutionnelle, 128/2001, cité par J. M. VÁzquez García-Peñuela, « Law and Religion in the Workplace in Spanish Legal System», in M. Rodriguez Blanco (ed.), op. cit., p. 366.

36. Ibid., p. 381.

37. Accord entre l'Italie et le Saint-Siège du 16 février 1984.

38. Le diritto ecclesiastico est le droit italien appliqué aux confessions religieuses et à leurs membres.

39. V. par ex. art. 5, 6, 7 et 13 de l'Accord entre l'Église évangélique luthérienne et l'Italie du 20 avril 1993. 
ministres du culte ne sont pas toujours détaillées. La tradition italienne en la matière fait montre d'une plus grande retenue par rapport à la neutralité de l'État et à l'autonomie des confessions religieuses. Le seul critère retenu est celui de l'accréditation par l'autorité religieuse. Ainsi, l'article 3 de la loi du 8 mars 1989 publiant l'accord avec l'Union des communautés juives italiennes indique que cette dernière nomme les rabbins conformément aux statuts de l'union et leur délivre un certificat. Ce document les autorise à célébrer des mariages religieux produisant des effets civils, à occuper des fonctions d'aumôniers dans les hôpitaux et les établissements pénitentiaires et l'armée et enfin à bénéficier d'un accès au Fonds spécial de prévoyance et d'assurance des ministres du culte. La loi du $1^{\text {er }}$ avril 1995 publiant l'accord avec l'Union chrétienne évangélique baptiste d'Italie constate qu'il existe au sein de cette communauté une pluralité de fonctions de ministres du culte et laisse le soin à cette confession religieuse d'en dresser la liste. Les agents religieux y figurant peuvent occuper les fonctions d'aumôniers et bénéficier de prestations sociales spécifiques. Par contre, l'accord avec les mormons de 2012 énumère les différentes fonctions de ministres du culte de cette confession: président de pieu et président de district, évêque et président de branche, président de temple et président de mission. Enfin, les ministres du culte hindouistes sont ceux cités par les statuts annexés à l'entente et qui sont accrédités par la confession religieuse. Les autorités publiques sont pragmatiques. Les fonctions religieuses sont dans la mesure du possible énoncées, à défaut l'Administration se contente d'une liste de noms. Mais dans tous les cas, l'agent cultuel doit être accrédité par les autorités de son culte.

L'ensemble des ministres du culte, qu'ils relèvent en tant que clercs de la religion catholique ou d'une religion ayant conclu une entente avec l'État, sont certes affiliés au régime général de la sécurité sociale, mais avec une exclusion du contrat de travail. Ce ne sont pas des travailleurs ordinaires dont l'objectif est de gagner un salaire, mais des individus dont l'activité est profondément liée à un engagement religieux ${ }^{40}$. Partant, les ministres du culte, et plus particulièrement les prêtres catholiques, ne perçoivent pas un salaire en contrepartie de leur ministère. La Cour suprême italienne a considéré dans une décision de $1996^{41}$ que les prêtres œuvraient pour le bien de l'homme dans l'intérêt de la nation et que l'argent versé pour leur entretien

40. S. Ferrari, «Italy», in G. Robbers and C. Durham (eds), op. cit., p. 207 et V. Pacillo, «Law and Religion in the Workplace: the Italian report», in M. RodRiguez BLANCo (ed.), op. cit., p. 257-258.

41. Cour de cassation, 27 mai 1996, $n^{\circ} 4871$. 
s'apparentait à une aide sociale et ne relevait pas du salariat ${ }^{42}$. Le poids du modèle catholique et du droit canonique reste là également très prégnant.

\subsection{LES MINISTRES DU CULTE ENTRE LIBERTÉ D'ORGANISATION DES CULTES ET RESPECT DES DROITS FONDAMENTAUX DE LA PERSONNE}

Aux Pays-Bas, la liberté d'organisation des cultes est garantie par l'article 6 de la Constitution et par l'article 2.2 du Code civil qui dispose que les Églises et leurs démembrements ont la personnalité juridique. Ils sont régis par leurs propres statuts tant qu'ils n'entrent pas en conflit avec la loi. Aux termes de ce texte, les religions ont la capacité de fixer les relations de travail avec les ministres du culte et certains employés des entreprises religieuses. L'exclusion du contrat de travail n'est pas automatique, les religions ayant le choix entre contrat de travail et exclusion du contrat. Mais dans tous les cas de figure, il revient aux juridictions lorsqu'elles sont saisies à l'occasion d'un contentieux de déterminer s'il existe une relation de travail au regard des conditions requises pour son existence ${ }^{43}$. Les décisions des autorités religieuses peuvent faire l'objet d'un recours devant les juridictions nationales lorsque le plaignant considère qu'elles n'ont pas été prises de bonne foi et que la procédure n'a pas été respectée. Les Églises et religions s'organisent librement, mais leurs décisions peuvent dans tous les cas être contestées devant les juridictions civiles conformément à l'article 17 de la Constitution ${ }^{44}$. Cette relative souplesse permet de prendre en considération les différences entre les cadres des diverses religions. Le clergé chrétien n'a par exemple en règle générale pas de contrat de travail, alors que la majorité des imams en détiennent un.

\section{CONCLUSION}

Cette étude sur les statuts des ministres des cultes dans les États de l'Union européenne pourrait se conclure sur le constat d'une très grande diversité de cas écartant toute tentative de comparaison. Il est au contraire possible de

42. En application des accords de 1984, les prêtres catholiques en fonction dans les paroisses et l'administration diocésaine ont droit à une rémunération dont le montant est fixé par la conférence épiscopale.

43. S. van Bisterveld, «Law and Religion in the Workplace: The Netherlands », in M. Rodriguez Blanco (ed.), op. cit., p. 319-321.

44. «Nul ne peut être distrait contre son gré du juge que la loi lui assigne.» 
dégager des lignes de convergences adossées notamment à la jurisprudence de la Cour européenne des droits de l'homme. Il apparaît nettement que les régimes nationaux des cultes, souvent forgés au cours de longs développements historiques, sont dorénavant soupesés et remis en question par le législateur et le juge. Cette évolution est surtout perceptible dans les pays où les clercs constituaient une forme de corporation comme en Angleterre. Si les titulaires d'un bénéfice y sont encore préservés grâce à la complexité de cette architecture où le clerc anglican est durante munere un quasi-propriétaire du bien dont les revenus lui sont affectés, les titulaires d'un office rémunéré sont dans certains cas dorénavant assimilés à des salariés. Mais toutes les poches de résistance n'ont pas disparu. Les États concordataires du sud de l'Europe fortement marqués par le droit canonique maintiennent un modèle catholique adossé à une survalorisation du principe d'autonomie des cultes qui est sans aucune nuance étendu à toutes les autres confessions religieuses. Cette situation, qui entraîne une non-intervention du juge civil en cas de conflit, ne joue pas en faveur de la protection des agents cultuels et est sévèrement critiquée par la doctrine. Mais dans la majorité des États, les textes et la jurisprudence tendent au contraire à distinguer le spirituel du temporel et à mettre en œuvre des mécanismes pour garantir un juste procès et éviter l'arbitraire des autorités religieuses. De plus, l'exclusion du contrat de travail ne s'impose plus comme un principe, mais devient plutôt une option. Les tribunaux supérieurs recommandent de se défaire du poids de la tradition et de prendre en considération les éléments qui permettront ou non de déterminer l'existence d'une relation de travail. 